\title{
Microbiological surveillance and antimicrobial stewardship minimise the need for ultrabroad-spectrum combination therapy for treatment of nosocomial infections in a trauma intensive care unit: An audit of an evidence-based empiric antimicrobial policy
}

\author{
Y Ramsamy, ${ }^{1,2}$ MB ChB; D J J Muckart, ${ }^{3}$ FRCS, MMSc Crit Care (SA); K S S Han, ${ }^{4,2}$ MB BS, FC Path (Microbiology), MMed Microbiology, \\ DTMH, PDIC \\ ${ }^{1}$ Department of Medical Microbiology, Nelson R Mandela School of Medicine, University of KwaZulu-Natal, Durban, South Africa \\ ${ }^{2}$ National Health Laboratory Service, KZN Academic Complex, KwaZulu-Natal, South Africa \\ ${ }^{3}$ Department of Surgery, Nelson R Mandela School of Medicine, University of KwaZulu-Natal, Durban, South Africa \\ ${ }^{4}$ Department of Medical Microbiology, Nelson R Mandela School of Medicine, University of KwaZulu-Natal, Durban, South Africa
}

Corresponding author: Y Ramsamy (yogandree@gmail.com)

\begin{abstract}
Background. Nosocomial infections are a major cause of morbidity in the critically injured, and the incidence of resistant strains of bacteria is increasing. Management requires a strategy that achieves accurate empiric cover without antibiotic overuse - a goal that may be achieved by surveillance and antibiotic stewardship.

Objectives. With the aim of minimising the use of empirical ultrabroad-spectrum combination antimicrobial prescriptions and reducing bacterial resistance, the level I Trauma Intensive Care Unit (TICU) at Inkosi Albert Luthuli Central Hospital (IALCH) in Durban employs stewardship and an antimicrobial policy based on surveillance. This study was undertaken with three aims: (i) to describe the spectrum and sensitivities of nosocomial pathogens in a level I TICU; (ii) to ascertain, based on surveillance data, how frequently initial empiric choice of antimicrobials was correct; and (iii) to determine how frequently ultrabroad-spectrum antimicrobials were prescribed and were actually necessary.

Methods. Over a 12-month period, all critically injured patients who underwent mechanical ventilation in the TICU were identified from a prospectively gathered database. Information regarding every specimen submitted to the National Health Laboratory Services (NHLS) situated at IALCH was extracted from the laboratory computer database. For each patient, bacterial isolates and antimicrobial susceptibility were identified using standard laboratory techniques. Empiric prescriptions for presumed nosocomial sepsis were identified from the hospital's computerised patient record system and compared with culture results. Acinetobacter species were regarded as colonisers and treatment not offered unless this was the sole isolate in the presence of signs of severe sepsis.

Results. Of 227 patients, 106 (46.6\%) had 136 culture-positive isolates with a total of 323 pathogens (201 Gram-negative, 119 Grampositive, 3 Candida albicans). There were 19 species of Gram-negative pathogens, of which $56 \%$ comprised Enterobacteriaceae. Extended spectrum beta-lactamase (ESBL) production was found in 6/31 (19\%) Escherichia coli coli and 6/24 (25\%) Klebsiella isolates. Staphyloccocal species accounted for $60 \%$ of the Gram-positive isolates, of which 18 were methicillin-resistant Staphylococcus aureus (MRSA). All Candida isolates were sensitive to fluconazole. One hundred and one empiric and 14 directed prescriptions were issued. Despite positive cultures, antimicrobials were not prescribed for 21 patients who had no evidence of sepsis. Excluding multidrug-resistant Acinetobacter isolates, there were 87 (93.5\%) appropriate and 6 (6.5\%) incorrect prescriptions. Ultrabroad-spectrum combination therapy (U-bSCT) was employed for 11 patients but was necessary in only 2 .

Conclusions. When combined with regular bacterial surveillance, antimicrobial stewardship allows accurate empiric antimicrobial prescription with minimal need for ultrabroad-spectrum combination therapy. This policy can potentially reduce the emergence of multidrug-resistant pathogens, precluding the need for broad-spectrum antimicrobials and the attendant problems of overuse.
\end{abstract}

S Afr Med J 2013;103(6):371-376. DOI:10.7196/SAMJ.6459

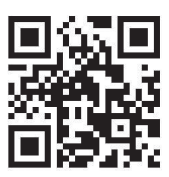

Multidrug-resistant organisms are an emerging threat in South Africa (SA). ${ }^{[1]}$ Inappropriate prescriptions of broad-spectrum antimicrobials, multiple drug combinations, prolonged treatment and the lack of de-escalation are the main reasons, and Mendelson et al. warn that we are on course towards an era of untreatable bacterial infections. ${ }^{[2]}$ Indiscriminate prescribing is common in SA intensive care units (ICUs) ${ }^{[3]}$ driven by the fear that selecting the wrong antimicrobial to treat nosocomial infections will result in treatment failure and an increased mortality rate. ${ }^{[4,5]}$ Ultrabroad-spectrum combination therapy (U-bSCT), whereby the initial empiric choice covers all possible pathogens, has been used injudiciously, with as many as 10 anti-infectives having been prescribed simultaneously. This policy is hazardous and promotes the development of multidrugresistant (MDR) pathogens. ${ }^{[6]}$

Strategies to minimise the development of resistance (such as class restriction, ${ }^{[7]}$ antibiotic cycling ('crop rotation') ${ }^{[8]}$ and antimicrobial stewardship ${ }^{[9]}$ ) have been proposed. Unfortunately, 
class restriction of specific agents and antibiotic cycling has had little effect and, with time, have demonstrated an increase in resistance to alternative drugs. ${ }^{[8-10]}$ The most effective strategies are strict infection control measures and optimising both prophylactic and therapeutic antimicrobial use by surveillance, establishing a protocol, education and stewardship. ${ }^{[2,11]}$ An effective empiric protocol based on knowledge of local microbiological patterns is essential, as is defining those risk factors that need ultrabroadspectrum combination cover.

Our trauma intensive care unit (TICU) subscribes to stewardship and employs an empiric antimicrobial policy based on surveillance. The purpose of this study was threefold: (i) to describe the spectrum of nosocomial pathogens in a level 1 TICU; (ii) to ascertain, on the basis of monthly surveillance, how frequently the initial empirical choice of antimicrobials was correct; and (iii) to determine whether U-bSCT was warranted and, when used, how frequently it was actually necessary.

\section{Patients and methods}

This study was approved by the Bioethics Committee of the University of KwaZulu-Natal (BE 132/010) and was performed from 1 January to 31 December 2009 in the TICU at the Inkosi Albert Luthuli Central Hospital (IALCH) a tertiary/quaternary public service institution in Durban. The TICU contains 10 beds used exclusively for critically injured patients, providing support for both adults and children regardless of age. The study included all patients who underwent mechanical ventilation. The staff comprise 3 fulltime specialists trained in trauma surgery and critical care who decide admissions and make all treatment decisions.

All patients admitted to the TICU who underwent mechanical ventilation during the study period were entered into a computerised database. Information regarding every specimen submitted from patients in the database to the National Health Laboratory Services (NHLS) situated at IALCH was extracted from the laboratory computer database. Processing of specimens, identification of pathogens and antimicrobial susceptibility testing was carried out as per standard NHLS operating procedures. ${ }^{[12]}$ All laboratory results were correlated with the patients' clinical condition and with adjunct investigations such as radiology, white cell counts and quantified procalcitonin levels, which were obtained from the hospital's Medicom information system. From the computerised prescription database, details of all patients who received antimicrobials were extracted and the selected empiric therapy analysed against bacterial sensitivity.

Ventilator-associated pneumonia (VAP) was defined as pneumonia occurring in a patient within 48 hours or more after intubation with an endotracheal or tracheostomy tube and which was not present before admission. The diagnosis was supported by presence of new pyrexia $>38.4^{\circ} \mathrm{C}$, changes on chest auscultation, new infiltrates on chest radiology, purulent endotracheal aspirate (ETA), a rise in white cell count, and elevated procalcitonin. Early-onset VAP was defined as occurring within 72 hours, and late-onset VAP beyond 72 hours following tracheal intubation. If multiple organisms were demonstrable in tracheal aspirates and VAP was suspected clinically, the choice of antimicrobial was made on the basis of the pathogen deemed to be most probably responsible. In this setting, Acinetobacter baumannii and other related species were regarded as colonisers; the organisms were usually multidrug-resistant and therapy was not selected on the basis of their sensitivities.

Vascular catheter-related bloodstream infection was defined by the clinical features of sepsis, growth of the same organism from peripheral blood and blood culture aspirated from either the intravenous (IV) catheter or a catheter segment, the absence of any other possible source of the infection, and resolution of the signs of sepsis within 24 hours of IV removal in the absence of antimicrobial or antipyretic therapy. A semiquantitative culture method for identifying IV catheter infection was employed to diagnose sepsis. Such bloodstream infection was managed by line removal alone unless the skin entrance site appeared septic. Replacement catheters were always inserted at a new site.

Urinary tract infection was defined by association of 2 of the following criteria: clinical signs of sepsis, pyuria $\geq 10$ white blood cells (WBCs) $/ \mathrm{mm}^{3}$, urine culture of $10^{5}$ colony-forming unit (CFUs)/ $\mathrm{ml}$, abnormal microscopy of urine, and the presence of nitrites on dipstick testing. The diagnosis of surgical site infection (SSI) was based on clinical examination, operative findings and microbiological analysis of specimens.

The antimicrobial protocol, established in conjunction with the Department of Medical Microbiology, for surgical prophylaxis and treatment of community- and hospital-acquired infections employed by the TICU is shown in Table 1. U-bSCT was not used as a matter of routine, and was instituted only if patients failed to respond within 48 hours to the first-line choice for suspected nosocomial infection, or if a recurrent episode of nosocomial sepsis was complicated by a sudden deterioration in organ function.

\section{Results}

During the study period, 227 patients were managed in the TICU: 144 with motor vehicle-related trauma (pedestrians 67, passengers 47, drivers 30 ), 36 for gunshot wounds, 26 non-vehicular blunt injury, and 21 stab wounds. There were $174(76.7 \%)$ males and $53(23.3 \%)$

Table 1. Empirical policy for antimicrobial prophylaxis and therapy based on surveillance and co-existent organ dysfunction

\begin{tabular}{lll}
\hline & Prophylaxis & Therapy \\
\hline $\begin{array}{l}\text { Community-acquired } \\
\text { Pneumonia }\end{array}$ & & $\begin{array}{l}\text { Amoxycillin/clavulanic } \\
\text { acid }\end{array}$ \\
Peritonitis & Cefoxitin & $\begin{array}{l}\text { Amoxycillin/clavulanic } \\
\text { acid + gentamicin } \\
\text { Cloxacillin or moxycillin/ } \\
\text { clavulanic acid }\end{array}$
\end{tabular}

Hospital-acquired

Pneumonia

Piperacillin/tazobactam + amikacin

Piperacillin/tazobactam + ciprofloxacin ${ }^{\dagger}$

Tertiary peritonitis

Piperacillin/tazobactam + amikacin

Piperacillin/tazobactam + ciprofloxacin ${ }^{\dagger}$

(Fluconazole is added for foregut injury)

Catheter-related Cloxacillin

insertion site infection

Ultrabroad therapy

Meropenem + vancomycin + fluconazole $^{\ddagger}$

*If severe contamination.

If acute kidney injury present

If risk factors for fungal sepsis are present, which include penetrating foregut injury, repeated laparotomies, and prior treatment for nosocomial bacterial infections on more than occasions. 
females, with a mean age of 29.2 years (range 2 - 76). The median injury severity score (ISS) was 25 (IQR 16 - 29).

Of the 227 admissions, 106 (46.7\%) yielded a total of 323 positive cultures of which 201 (62\%) were Gram-negative, 119 (37\%) Grampositive, and $3(1 \%)$ cultured Candida albicans. There were 19 different species of Gram-negative (Fig. 1) and 10 of Gram-positive (Fig. 2) organisms. Of the Escherichia coli isolates, 6 (19\%) were

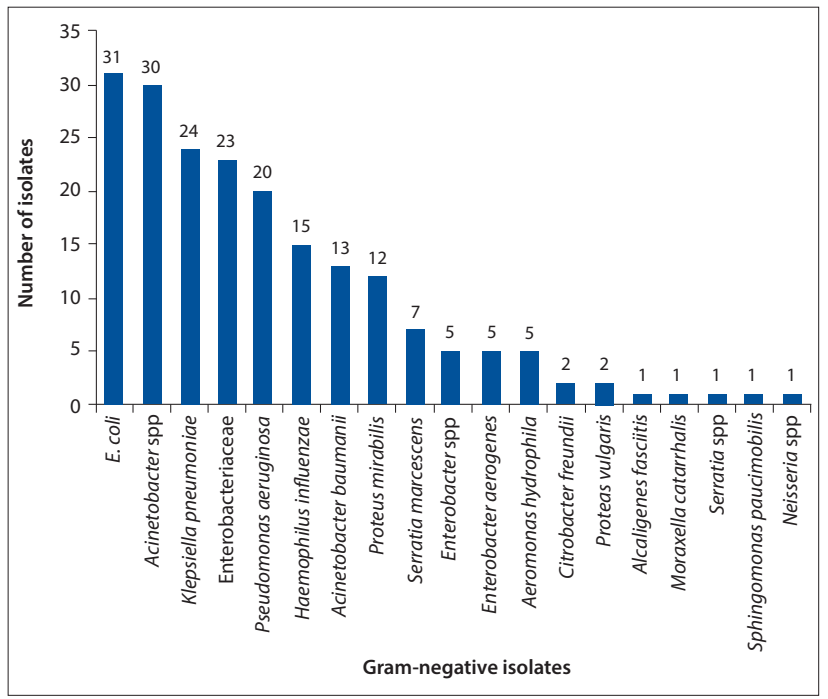

Fig. 1. Frequency and species of 201 Gram-negative isolates.

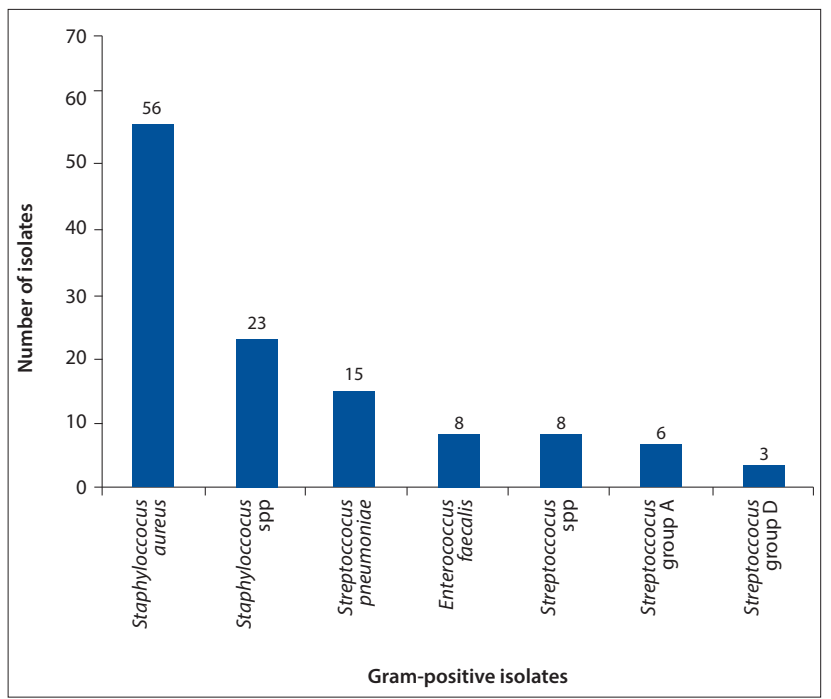

Fig. 2. Frequency and species of most common Gram-positive isolates. extended spectrum ß-lactamase (ESBL) positive, 19 (62\%) were ESBL negative, and 6 (19\%) were not tested. Routine testing of ESBLs was not performed if the organism was sensitive to amoxicillin/clavulanic acid. There were also 6 (25\%) ESBL positive isolates of Klebsiella pneumoniae, $12(50 \%)$ were ESBL negative, and $6(25 \%)$ were not tested for the reasons outlined above. The 18 staphylococcal isolates that were reported as methicillin resistant arose from only 7 patients, 4 of whom had been transferred from other institutions.

Table 2 illustrates the distribution of positive cultures by specimen site, the Gram type of organism and number of patients. Table 3 shows the most common specific organisms isolated. Antimicrobial susceptibility testing was carried out on the various isolates to establish the accuracy of empiric treatment. All isolates were tested for their unique antimicrobial susceptibility patterns, which are illustrated in Tables 4 and 5 for the most common pathogens.

There were 101 empirical antimicrobial prescriptions prior to receipt of culture results, of which 90 (89\%) involved first-line therapy consisting of piperacillin/tazobactam with either amikacin or ciprofloxacin, and 11 (11\%) U-bSCT prescriptions using meropenem and vancomycin with the addition of fluconazole in 4 . Fourteen directed prescriptions were issued according to culture results. In 21 instances, antimicrobials were not prescribed, despite positive cultures, owing to absence of clinical signs of sepsis (Fig. 3).

Of the 101 empiric prescriptions, 87 (86\%) were correct, based on organism susceptibility, while $14(14 \%)$ did not cover the isolated pathogens. Of the latter, there were 8 isolates of MDR Acinetobacter (7 endotracheal aspirates, 1 blood culture), of which 3 were treated with empiric U-bSCT and 5 received empiric first-line therapy. The former group of subjects all died, whilst the latter survived. Excluding these 8 Acinetobacter isolates as per unit policy there were therefore 87/93 (94\%) correct and 6/93 (6\%) incorrect empiric prescriptions (Fig 3). The latter consisted of inadequate cover for Candida species (2), ESBL-producing E. coli (2), ESBL-producing Klebsiella (1) and Serratia (1). Of the 11 empiric ultrabroadspectrum therapy prescriptions, 6 isolates were piperacillin/ tazobactam-sensitive, in 3 multidrug-resistant Acinetobacter was the primary pathogen isolated, while the remaining 2 consisted of ESBL-producing E. coli and ESBL-producing Klebsiella sensitive to ciprofloxacin and meropenem respectively. Therefore only 2 of the 11 prescriptions were appropriate. There were 25 positive culture isolates in the 21 patients who did not receive antimicrobials; tracheal aspirates accounted for $62 \%$, central venous catheter tips and surgical site specimens for $14 \%$ each, and blood cultures for $10 \%$. There were no deaths in this group of 25 , despite the absence of therapy.

Among the 227 admissions, there were 40 (18\%) deaths, of whom $16(40 \%)$ subjects had undergone antimicrobial treatment

Table 2. Distribution of positive cultures by specimen site

\begin{tabular}{llllll}
\hline Specimen & Gram-negative $\boldsymbol{n}(\%)$ & Gram-positive $\boldsymbol{n}(\%)$ & C. albicans $\boldsymbol{n}(\%)$ & Total $\boldsymbol{n}(\%)$ & Number of patients $^{*}$ \\
\hline ETA & $88(65)$ & $47(34)$ & $1(1)$ & $136(42.1)$ & 71 \\
SSI & $68(67)$ & $32(32)$ & $1(1)$ & $101(31.3)$ & 44 \\
CVC & $17(39)$ & $26(59)$ & $1(2)$ & $44(13.6)$ & 36 \\
Blood & $21(66)$ & $11(34)$ & & $32(9.9)$ & 24 \\
Urine & $7(70)$ & $3(30)$ & $3(1)$ & $10(3.1)$ & 10 \\
Total & $201(62)$ & $119(37)$ & & $323(100)$ &
\end{tabular}

ETA = endotracheal aspirate; $\mathrm{SSI}=$ surgical site infection; $\mathrm{CVC}=$ central venous catheter ${ }^{*}$ Some patients had more than one site of sepsis. 
Table 3. The most common isolates $(\geq 15)$ per specimen site

\begin{tabular}{|c|c|c|c|}
\hline Pathogen $(n)$ & $\begin{array}{l}\text { Lower respiratory tract infection } \\
n(\%)\end{array}$ & $\begin{array}{l}\text { Surgical site infection } \\
n(\%)\end{array}$ & $\begin{array}{l}\text { Bloodstream infection }{ }^{*} \\
n(\%)\end{array}$ \\
\hline \multicolumn{4}{|l|}{ Gram-negative } \\
\hline E. coli $(31)$ & $8(25.8)$ & $17(54.8)$ & $4(12)$ \\
\hline K. pneumoniae (24) & $13(54.1)$ & $6(25)$ & $5(20.8)$ \\
\hline Acinetobacter spp. (30) & $17(56.6)$ & $6(19.3)$ & $7(23.3)$ \\
\hline E. cloacae (23) & $6(26)$ & $6(26)$ & $8(34.7)$ \\
\hline P. aeruginosa (20) & $7(35)$ & $13(65)$ & $0(0)$ \\
\hline A. baumannii (13) & $6(46)$ & $3(23)$ & $4(30.7)$ \\
\hline H. influenzae (15) & $13(86.6)$ & $1(6.6)$ & $1(6.6)$ \\
\hline \multicolumn{4}{|l|}{ Gram-positive } \\
\hline S. aureus (56) & $33(58.9)$ & $12(21.4)$ & $11(19.6)$ \\
\hline S. spp. (23) & $1(4.4)$ & $0(0)$ & $22(95.6)$ \\
\hline S. pneumoniae (15) & $10(66)$ & $1(6.6)$ & $4(26.6)$ \\
\hline
\end{tabular}

Table 4. Antimicrobial susceptibility of the more common ( $\geq 15$ isolates) Gram-negative organisms

\begin{tabular}{|c|c|c|c|c|c|c|}
\hline \multirow[b]{2}{*}{ Organism (number of isolates) } & \multicolumn{6}{|c|}{ Antimicrobial sensitivity $n(\%)$} \\
\hline & Mer & Pip/taz & Amik & Cipro & Genta & Amoxy-clav \\
\hline E. $\operatorname{coli}(31)$ & $30(97)^{\dagger}$ & $28(90)$ & $28(90)$ & $26(84)$ & $29(94)$ & $14(45)$ \\
\hline Acinetobacter spp. (30) & $3(10)$ & $4(13)$ & $13(43)$ & $3(10)$ & $4(13)$ & $0(0)$ \\
\hline K. pneumoniae (24) & $22(92)^{*}$ & $19(79)$ & $20(83)$ & $18(75)$ & $16(66)$ & $14(58)$ \\
\hline E. cloacae (23) & $22(96)^{\dagger}$ & $21(91)$ & $21(91)$ & $19(83)$ & $18(78)$ & $0(0)$ \\
\hline$P$. aeruginosa (20) & $19(95)^{\dagger}$ & $\begin{array}{l}11(55)-S \\
9(45)-I\end{array}$ & $19(95)$ & $15(75)$ & $14(70)$ & $0(0)$ \\
\hline H. influenzae (15) & & & & $14(93)^{\dagger}$ & & $15(100)$ \\
\hline A. baumanii (13) & $3(23)$ & $2(15)$ & $9(69)$ & $6(46)$ & $2(15)$ & $0(0)$ \\
\hline P. mirabilis (12) & $12(100)$ & $10(83)$ & $12(100)$ & $10(83)$ & $11(92)$ & $10(83)$ \\
\hline
\end{tabular}

Table 5. Antimicrobial susceptibility of the more common ( $\geq 15$ isolates) Gram-positive organisms

\begin{tabular}{|c|c|c|c|c|c|c|c|}
\hline \multirow[b]{2}{*}{ Organism (number of isolates) } & \multicolumn{7}{|c|}{ Antimicrobial sensitivity $n(\%)$} \\
\hline & Amp & Clinda & Clox & Eryth & Genta & Pen & Vanco \\
\hline S. aureus (56) & $9(16)$ & $40(71)$ & $38(68)$ & $40(71)$ & $35(62)$ & $6(11)$ & $56(100)$ \\
\hline Staphylococcus spp. (23) & $5(22)$ & $13(56)$ & $8(35)$ & $9(39)$ & $7(30)$ & $3(13)$ & $22(96)^{*}$ \\
\hline S. pneumoniae (15) & $10(67)$ & $2(13)$ & $0(0)$ & $8(53)$ & $0(0)$ & $14(93)$ & $2(13)^{\dagger}$ \\
\hline
\end{tabular}

for presumed nosocomial sepsis. Seven (44\%) of the 16 deaths were in the incorrect treatment group, the isolates revealing MDR Acinetobacter in 3, Candida in 3 and ESBL-producing E. coli in 1. In these individual patients, $C$. albicans, susceptible to fluconazole, was isolated on day 3 from a tracheal aspirate, on day 5 from a central venous catheter tip, and on day 7 from abdominal fluid; and $E$. coli on day 3 from a tracheal aspirate and blood culture. All patients in whom
Candida was isolated had abdominal gunshot wounds with hollow visceral injuries; in 1, Candida was cultured from abdominal fluid at relaparotomy despite administration of prophylactic fluconazole for 48 hours. The patient in whom E. coli was cultured had sustained severe traumatic brain and chest injuries and multiple compound fractures (managed by decompressive craniectomy and damage control orthopaedic surgery). 


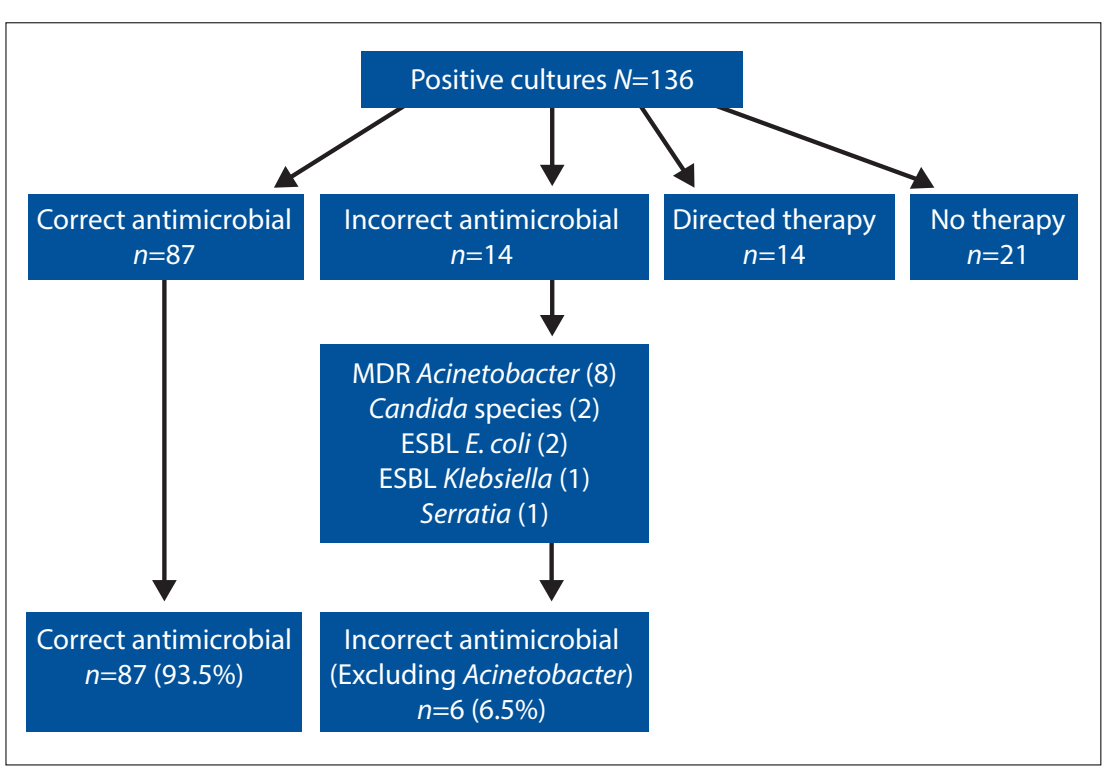

Fig. 3. Correct, incorrect and directed prescriptions for nosocomial sepsis.

There were 24 (20\%) deaths in 121 patients who did not suffer nosocomial sepsis, and 16 (15\%) in the 106 in whom positive isolates were recovered. Of the former, 11 deaths occurred either during resuscitation or emergency surgery, these individuals having not reached ICU. Excluding these early deaths, there was no significant difference in mortality between those with or without nosocomial sepsis $(p=0.55)$.

\section{Discussion}

In this study, Gram-negative organisms of the Enterobacteriaceae family predominated, closely followed by Pseudomonas. Grampositive species from which Staphylococcus aureus was isolated most frequently were less common. Although the flora may be shown to be similar, the extent and specific detail of bacterial resistance to antimicrobials varies considerably in reported series. While intrinsic genetic coding accounts for some instances of resistance, the most common cause is the overuse of broad-spectrum agents and selective pressure on the micro-organism. The pathogens causing most concern are more easily remembered by the mnemonic ESKAPE, i.e. Enterococcus faecium, $S$. aureus, K. pneumoniae, Acinetobacter spp., Pseudomonas aeruginosa, and Enterobacter spp. ${ }^{[13]}$ The most common mechanism of resistance is the production of ESBLs by Enterobacteriaceae, especially $K$. pneumoniae and E. coli. This may arise in almost $50 \%$ of Klebsiella isolates, ${ }^{[14]}$ and their prevalence therefore dictates empiric antimicrobial choice. The low prevalence of MRSA in our cohort reflects the lack of exposure to antimicrobials in the general population; we have yet to encounter vancomycin resistance, hence the use of this glycopeptide as first-line therapy. Not one patient died from this infection despite the fact that no therapy was offered to 4 of the 7 patients. It has recently been suggested that this pathogen may not confer direct attributable mortality. ${ }^{[15]}$

Fungal infections are of increasing importance in ICUs, ${ }^{[16]}$ and in this study possibly accounted for 3 deaths among those offered inappropriate therapy. Candida is a normal commensal of the stomach and proximal intestine and, although we cannot present hard evidence, our experience of treating penetrating abdominal trauma with foregut injuries caused by gunshot wounds, indicates that prophylaxis for Candida is warranted, especially in those requiring ICU management. Establishing the diagnosis of invasive candidiasis may be difficult, and the burden of HIV infection in SA, with the potential for Candida overgrowth, adds further credence to this premise. Failure to provide fungal cover constitutes an act of omission in our view.

It is the policy of the TICU not to treat MDR A. baumannii or related species if occurring in a mixture of flora. There are several evidenced-based reasons for this policy: firstly, Acinetobacter is a long-term coloniser and can persist on a variety of inanimate surfaces for prolonged periods of time; secondly, Acinetobacter species have historically been considered organisms of low virulence and pathogenicity, and attributable morbidity and mortality has been an ongoing debate for the past 30 years, chiefly because of the difficultly of differentiating colonisation from infection. ${ }^{[17]}$ Recent work, however, suggests that mutant strains possess specific capsular proteins that confer increased virulence and resistance to the effects of complement in human serum and ascitic fluid; ${ }^{[18]}$ and thirdly, most Acinetobacter species are inherently resistant to all but a few antimicrobials, and embarking on therapy would necessitate the use of U-bSCT and the risk of treating colonisation rather than true infection, which in turn would lead to emergence of other MDR organisms. In addition, Acinetobacter has the ability to develop resistance even during directed therapy, ${ }^{[17]}$ and the isolation of subpopulations that are resistant to colistin is of serious concern. Although ethically debatable, this practice conforms to the principle of social justice over autonomy and complements the view of Mendelson et al. ${ }^{[1]}$ that inappropriate and unnecessary prescriptions of broad-spectrum antimicrobials may ultimately result in an era of untreatable bacterial infections.

Excluding Acinetobacter from the empiric equation, our choice of antimicrobials was correct in more than $90 \%$ of instances, indicating that frequent surveillance and stewardship promote a more rational and restricted policy of antimicrobial use and an infrequent resort to U-bSCT. It should be noted that protagonists of such therapy (that provides antimicrobials effective against ESBL-producing Gram-negatives, MRSA and Candida) cite improved outcomes and claim that resistance will not increase, provided that it is used for brief periods, and the spectrum narrowed once there is microbiological confirmation. We would suggest otherwise. Firstly, most patients would respond to such broad therapy and their clinicians continue with a full course of treatment with inevitable pressure on the microbial environment. Secondly, culture results may not be available within 48 hours, again resulting in protracted, but unnecessary, broad-spectrum therapy. Thirdly, given the natural history of the selection of resistant strains of bacteria, why should the response to frequent use of broadspectrum antimicrobials be any different in the future? Although the length of treatment might be abbreviated by de-escalation, total antimicrobial use will inevitably increase with detrimental consequences. ${ }^{[1]}$

In our cohort, only 11 prescriptions required U-bSCT, of which 9 were not warranted. Excluding Acinetobacter, regarding the deaths in patients not offered U-bSCT, there were 2 Candida isolates and 
one ESBL-producing E. coli. Of the former, as mentioned above, failure to immediately prescribe antifungal therapy accounted for the one potential attributable death. Given such data, we suggest that, with an active surveillance programme and empiric antimicrobial policy, blanket cover is rarely indicated. Kollef makes the additional salient statement that the empiric choice of antimicrobials should cover the most likely pathogens endemic to the specific location. ${ }^{[19]}$ It is important to note that the statement does not say all pathogens, and prescriptions cannot be based on uncommon organisms unless the situation dictates the need. To this end, antimicrobial stewardship is of paramount importance, especially in areas with frequent antimicrobial use. There are many challenges to successful stewardship, but the aims are education, prevention of antimicrobial overuse, and minimising the development of resistance. ${ }^{[20]}$ Pivotal to success are interested clinicians and microbiologists, knowledge of local resistance patterns, and an antimicrobial policy that optimises the choice, dose and duration of therapy.

Seventy years ago, Sir Alexander Fleming ${ }^{[21]}$ correctly predicted the development of antimicrobials when he stated, 'The intensive research which penicillin has stimulated may bring forth others as good, or even better, or the chemists may be able to modify the penicillin molecule so that its power is increased or its limitations are removed. There is still plenty of scope for further advance'. In the same book edited by Fleming, however, Porritt and Mitchell[ ${ }^{[22]}$ made the pessimistic prophesy: 'As with sulphonamides, the prophylactic use of penicillin will carry the risk of breeding resistant strains, a matter of serious practical importance that cannot be dismissed lightly'. Unfortunately, the indiscriminate use of the drugs that Fleming predicted has promoted the emergence of resistance to even the most broad-spectrum antimicrobials. While Fleming's prediction that there is scope for further advance in the manufacture of new antimicrobials undoubtedly held true in the first half of the 20th century, the same cannot be said for now. There are few, if any, new avenues ${ }^{[1,2]}$ and strict measures must be implemented to enforce infection control and curb indiscriminate and inappropriate antimicrobial prescribing. ${ }^{[2,3]}$

\section{References}

1. Brink A, Coetzee J, Clay C, et al. The spread of carbapenem-resistant Enterobacteriaceae in South Africa: Risk factors for acquisition and prevention. S Afr J Med 2012;102:599-601.

2. Mendelson M, Whitelaw A, Nicol M, Brink A. Wake up South Africa! The antibiotic 'horse' has bolted. S Afr J Med 2012;102:607-608

3. Paruk F, Richards G, Scribante J, et al. Antibiotic prescription practices and their relationship to outcome in South African intensive care units: Findings of the Prevalence of Infection in South African Intensive Care Units (PISA) Study. S Afr J Med 2012;102:613-616.

4. Wagh H, Acharya D. Ventilator associated pneumonia - An overview. British Journal of Medical Practitioners 2009;2(2):16-19.

5. Lynch JP. Hospital-acquired pneumonia. Risk factors, microbiology, and treatment. Chest 2001;119:373S-384S. [http://dx.doi.org/10.1378\%2Fchest.119.2_suppl.373S]

6. Fraimow HS, Tsigrelis C. Antimicrobial resistance in the intensive care unit: Mechanisms, 6. Fraimow HS, Tsigrelis C. Antimicrobial resistance in the intensive care unit: Mechanisms,
epidemiology, and management of specific resistant pathogens. Crit Care Clin 2011;27:163-205. [http://dx.doi.org/10.1016\%2Fj.ccc.2010.11.002

7. Rahall JJ, Urban C, Horn D, et al. Class restriction of cephalosporin use to control total cephalosporin resistance to nosocomial Klebsiella. JAMA 1998;280:1233-1237. [http://dx.doi. org/10.1001\%2Fjama.280.14.1233

8. Kollef MH. Is antibiotic cycling the answer to preventing the emergence of bacterial resistance in the intensive care unit? Clin Infect Dis 2006;43:S82-S88. [http://dx.doi.org/10.1086\%2F504484]

9. Lawrence KL, Kollef MH. Antimicrobial stewardship in the intensive care unit. Am J Resp Crit Care Med 2009;179:434-438. [http://dx.doi.org/10.1164\%2Frccm.200809-1394CP]

10. Brusselaers $\mathrm{N}$, Vogelaers $\mathrm{D}, \mathrm{Blot} \mathrm{S}$. The rising problem of antimicrobial resistance in the intensive care unit. Annals of Intensive Care 2011;1:47-53. [http://dx.doi.org/10.1186\%2F2110-5820-1-47]

11. Derensinski S. Principles of antimicrobial therapy in severe infections: Optimizing the therapeutic approach by use of laboratory and clinical data. Clin Infect Dis 2007; 45:S177-183.
a

12. NHLS. Q-pulse Standard Operating Procedures. Q-pulse 5/docs/active/MIC 1569v1, MIC 1587v1, 2. NHLS. Q-pulse Standard Operating Procedures. Q-pulse 5/docs/active/MIC 1569v
MIC 1840vi, MIC 1849v1. Johannesburg: NHLS, 2010 (accessed 15 December 2012).

13. Boucher HW, Talbot GH, Bradley JS, et al. Bad bugs, no drugs: no ESKAPE! An update from the Infectious Diseases Society of America. Clin Infect Dis 2009;48:1-12. [http://dx.doi. org/10.1086\%2F595011]

14. Dhillon RH, Clark J. ESBLs: A clear and present danger? Crit Care Res Pract 2012. Published online 6 June2011. [http://dx.doi.org/10.1155/2012/625170]

15. Rosenberger LH, LaPar DJ, Sawyer RG. Infections caused by multidrug resistant organisms are not associated with overall, all-cause mortality in the surgical intensive care unit: The 20,000 foot view. J Am Coll Surg 2012;214:747-755. [http://dx.doi.org/10.1016\%2F.j.jamcollsurg.2012.01.040]

16. Lepak A, Andes D. Fungal sepsis: Optimizing antifungal therapy in the critical care setting. Crit Care Clin 2011;27:123-147. [http://dx.doi.org/10.1016\%2Fj.ccc.2010.11.001]

17. Murray CK, Hospenthal DR. Acinetobacter infection in the ICU. Crit Care Clin 2008;24:237-248. [http://dx.doi.org/10.1016\%2Fj.ccc.2007.12.005]

18. Russo TA, Luke NR, Beanan JN, et al. The K1 capsular polysaccharide of Acinetobacter baumannii susa TA, Luke NR, Beanan JN, et al. The Kl capsular polysaccharide of Acinetobacter baumannil
strain 307-0294 is a major virulence factor. Infect Immun 2010;78:3993-4000. [http://dx.doi. org/10.1128\%2FIAI.00366-10]

19. Kollef MH. Hospital-acquired pneumonia and de-escalation of antimicrobial treatment. Crit Care Med 2001;29:1473-1475. [http://dx.doi.org/10.1097\%2F00003246-200107000-00029]

20. Doron S, Davidson LE. Antimicrobial stewardship. Mayo Clin Proc 2011;86:1113-1123. [http://dx.doi. org/10.4065\%2Fmcp.2011.0358]

21. Fleming A. History and Development of Penicillin. In: Penicillin. Fleming A, ed. London: Butterworth and Co., 1946:1-23.

22. Porritt AE, Mitchell GAG. Prophylactic Use of Penicillin. In: Penicillin. Fleming A, ed. London: Butterworth and Co., 1946:105-115.

Accepted 16 January 2013. 